MITSUBISHI ELECTRIC RESEARCH LABORATORIES

http://www.merl.com

\title{
Differentiable Spectrum Partition for Fractional Frequency Reuse in Multi-Cell OFDMA Networks
}

\author{
Weihuang Fu, Zhifeng Tao, Jinyun Zhang, Dharma Agrawal
}

TR2010-035 June 2010

\begin{abstract}
To mitigate inter-cell interference (ICI) and achieve higher spectrum efficiency, fractional frequency reuse (FFR) has been widely adopted by the next generation wireless systems, wherein different frequency reuse factors are applied to cell center and cell edge zones. In such conventional FFR, a contiguous radio spectrum is partitioned in a fixed fashion across all cells for edge and cell center zones. This approach evidently lacks the flexibility of inter-cell and/or intra-cell resource allocation adjustment and the capability of dealing with traffic load fluctuation and quality of service (QoS) requirements variations. This paper models the implementation of FFR in a multicell network environment and proposes a scheme called D-FFR that can adaptively partition radio spectrum in a distributed manner to achieve different FFR configuration among different cells. Resource demands and various inter-cell/intracell allocation constraints are accounted in D-FFR to enable differentiable inter-cell and/or intra-cell throughput and deliver higher spectrum efficiency. Analytical and extensive simulation results are provided in the paper to validate the effectiveness of our proposal scheme.
\end{abstract}

WCNC 2010

This work may not be copied or reproduced in whole or in part for any commercial purpose. Permission to copy in whole or in part without payment of fee is granted for nonprofit educational and research purposes provided that all such whole or partial copies include the following: a notice that such copying is by permission of Mitsubishi Electric Research Laboratories, Inc.; an acknowledgment of the authors and individual contributions to the work; and all applicable portions of the copyright notice. Copying, reproduction, or republishing for any other purpose shall require a license with payment of fee to Mitsubishi Electric Research Laboratories, Inc. All rights reserved.

Copyright (C) Mitsubishi Electric Research Laboratories, Inc., 2010

201 Broadway, Cambridge, Massachusetts 02139 



\title{
Differentiable Spectrum Partition for Fractional Frequency Reuse in Multi-cell OFDMA Networks
}

\author{
Weihuang $\mathrm{Fu}^{\dagger}$, Zhifeng Tao ${ }^{\star}$, Jinyun Zhang ${ }^{\star}$, and Dharma P. Agrawal ${ }^{\dagger}$ \\ ${ }^{\dagger}$ Center for Distributed and Mobile Computing \\ Department of Computer Science, University of Cincinnati, Cincinnati, OH 45221, USA \\ ${ }^{\star}$ Mitsubishi Electric Research Laboratories (MERL), Cambridge, MA 02139, USA \\ Email: fuwg@cs.uc.edu, tao@merl.com, jzhang@merl.com, and dpa@cs.uc.edu
}

\begin{abstract}
To mitigate inter-cell interference (ICI) and achieve higher spectrum efficiency, fractional frequency reuse (FFR) has been widely adopted by the next generation wireless systems, wherein different frequency reuse factors are applied to cell center and cell edge zones. In such conventional FFR, a contiguous radio spectrum is partitioned in a fixed fashion across all cells for edge and cell center zones. This approach evidently lacks the flexibility of inter-cell and/or intra-cell resource allocation adjustment and the capability of dealing with traffic load fluctuation and quality of service $(\mathrm{QoS})$ requirements variations. This paper models the implementation of FFR in a multicell network environment and proposes a scheme called D-FFR that can adaptively partition radio spectrum in a distributed manner to achieve different FFR configuration among different cells. Resource demands and various inter-cell/intracell allocation constraints are accounted in D-FFR to enable differentiable intercell and/or intra-cell throughput and deliver higher spectrum efficiency. Analytical and extensive simulation results are provided in the paper to validate the effectiveness of our proposed scheme.
\end{abstract}

\section{INTRODUCTION}

Multi-cell system [1] offers mobile subscribers (MSs) convenient and pervasive network access service through the worldwide deployment of base stations (BSs), where the coverage of neighboring BSs is required to be overlapped to secure seamless connection and handover procedures. On the other hand, inter-cell interference (ICI) [2] inevitably occurs due to the spatial reuse of frequency resource in multi-cell architecture, which is particularly serious at the coverage overlap from neighboring BSs.

Frequency reuse factor measuring spatial reuse frequency of a spectrum partition in a multi-cell network was designed to be constant in conventional systems. Appropriate factor selection ought to balance spectrum efficiency and ICI mitigation. However, non-uniformly distributed ICI makes the use of a constant reuse factor in a cell hard to achieve the optimal balance point. Benefiting from the operations of orthogonal frequency division multiple access (OFDMA) technology [3] on subcarries and OFDM symbols in both frequency and time domains, fractional frequency reuse (FFR) [4] can be implemented for the next generation wireless systems, such as IEEE 802.16 [5] and the third generation partnership project

Weihuang $\mathrm{Fu}$ worked on this study while visiting Mitsubishi Electric Research Lab, Cambridge, MA. long term evolution (3GPP LTE) [6] to mitigate ICI effectively, which was difficult to implement in a conventional cellular system. FFR splits a frequency band into multiple spectrum partitions and uses spectrum partitions on defined cell center or cell edge zones. Different frequency reuse factors are achieved by controlling the reuse distance of spectrum partitions on zones.

The divisions of a frequency band are identical and continuous in conventional FFR. The resource allocation for MSs in a given zone is within the corresponding spectrum partition. The achievable throughput of a cell zone is mainly governed by the amount of the resource in allocated partition [7]. With identical spectrum partition, optimal allocation is achieved only when the traffic loads are evenly distributed in the network, which is not always true in a practical system. Due to lack of flexibility in adjusting spectrum partition, performance of FFR could be seriously degraded with unevenly distributed traffic. Identical resource partitions made on zones further restrict the ability of FFR to deal with uneven traffic. Differentiated spectrum partitions are desirable in accommodating such a situation. However, optimal resource allocation in a multi-cell network has been shown to be NP-hard [8].

In this paper, we propose a scheme performing differentiated spectrum partition for FFR (D-FFR), derived from the characteristics and analysis on current FFR schemes: hard FFR, soft FFR A and soft FFR B. Our scheme is able to fully utilize the flexibility of OFDMA technology and enable each BS to have differentiable spectrum partition and resource allocation, providing effective ICI mitigation and elasticity in MAC layer quality of service (QoS). Extensive simulation results are also provided in comparing various FFR schemes and our proposed D-FFR.

The remainder of this paper is organized as follows. Model for FFR schemes is formed in Section II. Section III models our D-FFR and analyzes the allocation constraints. Flexible scheme is illustrated in Section IV. Section V provides the performance results with extensive simulations. Finally, the paper is concluded in Section VI.

\section{Modeling And Analysis}

Hexagonally deployed BSs on a two dimensional area $\mathbb{R}^{2}$ constitute multi-cells to provide network access service for MSs. The coverage of a single BS is modeled as a circle 


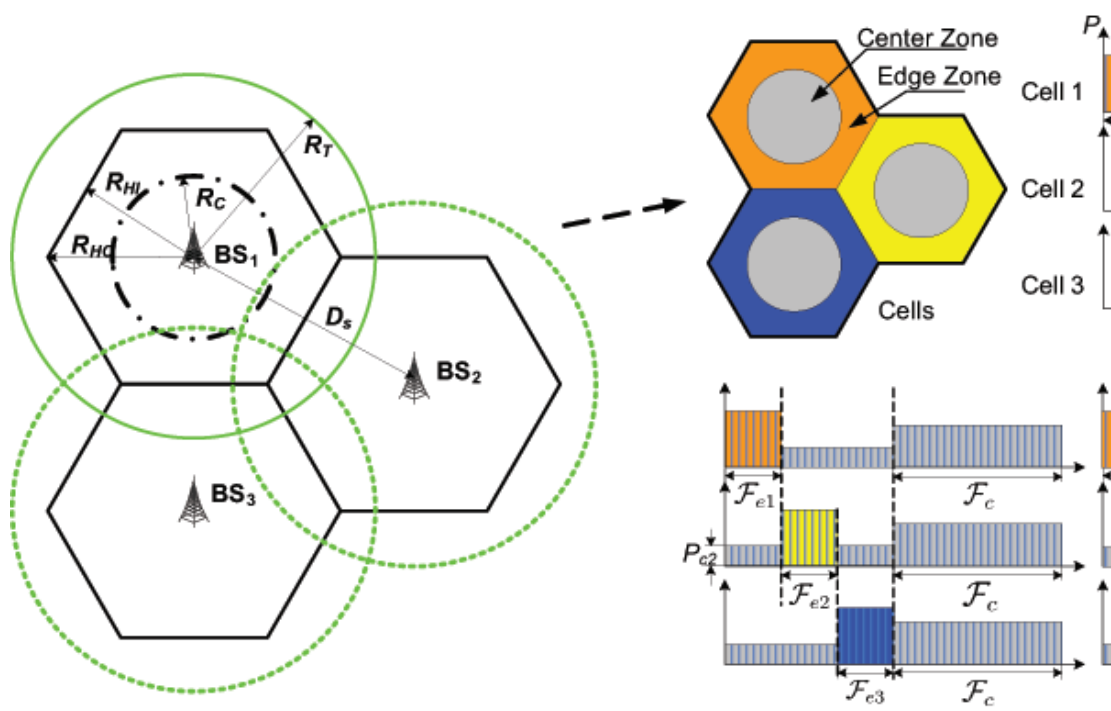

(b) Soft FFR A

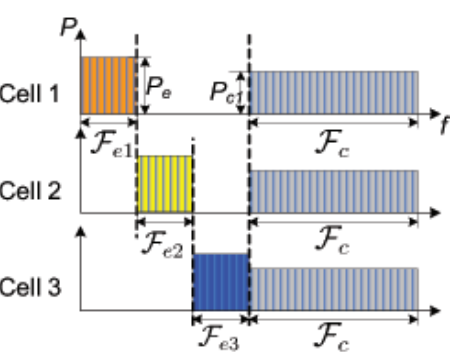

(a) Hard FFR

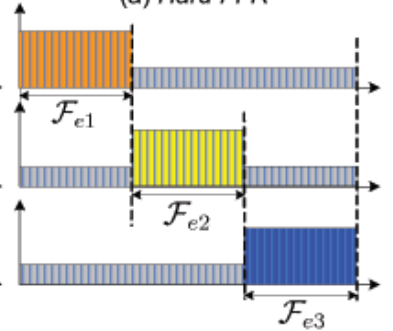

(c) Soft FFR B

Fig. 1: FFR schemes

centered at the BS with radius $R_{T}$, as shown in Fig. 1. Within the circle, MS is assumed to be able to decode the frame preamble sent by BS. Inter-BS distance, i.e., inter-site distance, is denoted by $D_{s}$. Different from the definition of BS coverage, BS cell is a hexagonal area centered at the BS, where hexagonal inradius $R_{H I}$ is $\frac{D_{s}}{2}$ and circumradius $R_{H C}$ is $\frac{D_{s}}{\sqrt{3}}$. BS cell is geometrically divided into edge zone $S_{e}$ and center zones $S_{c}$, illustrated by the simplified cells in Fig. 1, where cell center zone is the area around the serving BS and cell edge zone is the outer area of the cell. More specifically, the decision of zones made by BS depends on the diversity set reported by MSs. MS records the BS-IDs obtained from its received messages, forming a diversity set, and it is delivered for the serving BS to remember the MS location. FFR can be used either on cells (with omni-antenna) or on cell sectors (with directional antenna). To keep it simple, we discuss the case of cells.

OFDMA technology uses multiple orthogonal modulated subcarriers, which spreads over spectrum band $\mathcal{F}$. All OFDMA frames are synchronized and have the same time division duplex (TDD) for downlinks and uplinks. We focus on FFR performance in downlinks in this paper; however, the same mechanism is also applicable to uplinks. A group of subcarriers, in the term of a subchannel, can be assigned to MS. Two methods can be used to group subcarriers into subchannels: contiguous and distributed permutation. Contiguous permutation generates continuous frequency bandwidth with contiguous subcarriers. Distributed subcarriers use a permutation formula to organize subcarriers, which may be distributed on the frequency band. In this paper, subchannels formation can be either contiguous or distributed permutations. When using distributed scheme, a permutation formula is assumed to be the same at every BS. BS allocates downlink subchannels in set $\mathcal{F}=\{1, \ldots, F\}$ to MSs in its cell.

We use a factor to represent the level of frequency reuse.
For example, if reuse factor is three, it means the frequency is shared by three cells/sectors. The group of subchannels assigned to the cell edge zone is termed edge spectrum partition, which uses higher frequency reuse factor, and the group of subchannels allocated to the center zone is called center spectrum partition, which usually has frequency reuse factor of one. In other words, the spectrum partitions for the adjacent edge zones are non-overlapping and the spectrum partitions for the center zones of adjacent cells do overlap. To further reduce the interference among center zones, transmission power is also adjusted in the FFR based on different zones.

It should be noticed that the function of FFR is to divide frequency band and map the partitions to geometrical zones with different reuse factors. It is not directly in charge of resource allocation for MSs. The per-MS resource is further allocated by BSs within the given frequency partition allocated by FFR. Different from the per-MS allocation that may be changed by frames, FFR allocation lasts for relatively long time.

Based on the frequency reuse method, FFR can be categorized into two major types: hard FFR and soft FFR. Hard FFR uses dedicated center spectrum partition for center zone, while the center zone in soft FFR can reuse the frequency resource used by its adjacent edge zones. To mitigate any possible interference, the transmit power for such reuse is lower than that of dedicated center frequency resource. Soft FFR can be further divided into two types: soft FFR A and soft FFR B, depending on whether it has a dedicated center spectrum partition or not.

The spectrum allocation of hard FFR scheme is illustrated in Fig. 1(a), with frequency reuse factor three at edge zone and one at center zone. Center zones use identical center spectrum partition, while the MSs in the edge zones of adjacent cells are allocated different spectrum partitions. The vertical dotted lines in Fig. 1 indicate the separation of spectrum partitions, 
where the spectrum partitions of adjacent cell edges are nonoverlapping. The empty bandwidth in Fig. 1(a) is the spectrum partition not used by a cell, in order to satisfy edge spectrum partition allocation constraints. MSs in edge zone do not have the ICI from adjacent cells, which provides higher SINR than that in a reuse-1 scheme. The spectrum partition allocated for cell center zones is denoted by $\mathcal{F}_{c}$. The spectrum partitions allocated for three different edge zones are denoted by $\mathcal{F}_{e 1}$, $\mathcal{F}_{e 2}$, and $\mathcal{F}_{e 3}$, which are non-overlapping and the total amount of bandwidth of three spectrum partitions is complementary to the center bandwidth. The vertical axis indicates the transmit power. The subchannels for the edge zones use power $P_{e}$, which is higher than power $P_{c 1}$ used by the subchannels allocated for the center zones.

Figs. 1(b) and (c) illustrate soft FFR A and soft FFR B respectively. Different from hard FFR, the empty bandwidth is reused by soft FFR A for center zone with lower power $P_{c 2}$. It can provide higher center bandwidth resource usage; however, it may also cause more interference to the MSs in the center zone and the MSs in the edge zone of the adjacent cells. In soft FFR B, there is no dedicated center spectrum partition and the subchannels for center zone are overlapped with the adjacent cell edge subchannels.

In those FFR schemes, the spectrum partitions are fixed and identical, which have low spectrum efficiency to handle various traffic demands and QoS requirements in a practical system. In the following sections, we show the model and the algorithm to achieve differentiated spectrum partitions in FFR.

\section{D-FFR MODELING}

As the construction of spectrum partitions relies on the traffic loads in cells and zones, it needs to appropriately allocate subchannels and adaptively form spectrum partitions to achieve higher spectrum efficiency and lower ICI. In this section, we model the construction and the constraints in forming spectrum partitions.

A multi-cell network can be modeled by graph $G(V, E)$, where $u \in V$ denotes a cell and $u v \in E$ denotes the ICI relationship among cells. Edge $u v$ in set $E$ represents existence of strong ICI. For example, downlink signals from BS $u$ and downlink signals from BS $v$ both operate on the same radio resource at the same time. Downlink statistic traffic load of cell $u$ in a relatively long duration is denoted by $D^{(u)}$, which can be further divided into edge zone traffic load $D_{e}^{(u)}$, and center zone traffic load $D_{c}^{(u)}$. The allocation of subchannel $i$ to a partition can be denoted by a binary variable $A_{i}^{(u)}$. Transmit power is denoted by $P_{i}^{(u)}$, which takes value from three candidates: $P_{e}, P_{c 1}$, or $P_{c 2}\left(P_{e}>P_{c 1}>P_{c 2}\right)$. Subchannel allocation and transmit power are determined so as to satisfy the following constraints:

Edge zone allocation constraint: By using this constraint, adjacent cell edge zones are prevented from using the same subchannel. The constraint is expressed by:

$$
A_{i}^{(u)}+A_{i}^{(v)} \leq 1, \quad \forall i \in \mathcal{F}, \forall u v \in E .
$$

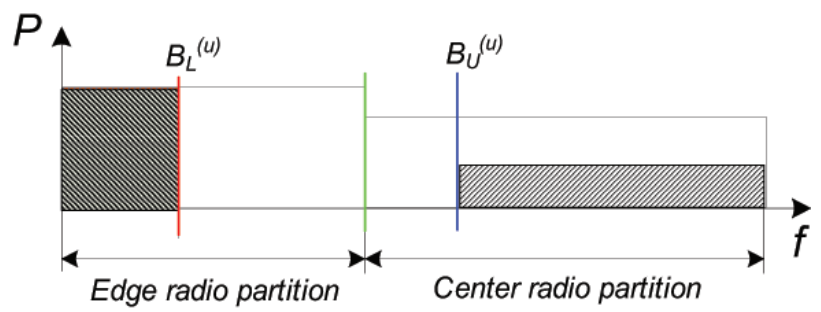

Fig. 2: Scheme illustration

Transmit power constraint: It constraints the transmit power used by adjacent cells on a same subchannel to prevent the subchannel from being excessively reused. Considering both hard FFR and soft FFR cases, the maximum transmit power of adjacent cells on a same channel should be no more than a pre-defined threshold $P_{e}+P_{c 2}$, which serves as an upper bound for radio reuse in adjacent cells:

$$
P_{i}^{(u)} A_{i}^{(u)}+P_{i}^{(v)}\left(1-A_{i}^{(v)}\right) \leq P_{m}, \forall i \in \mathcal{F}, \forall u v \in E,
$$

where $P_{m}=P_{e}+P_{c 2}$. If soft reuse is not allowed, we have $P_{m}=P_{e}$.

Edge spectrum partition reservation factor: Two bounds are set for the maximum and the minimum sizes of partitions. The first one, edge spectrum partition reservation factor $B_{L}^{(u)}$, is used to reserve radio resources for cell edge zone and serves as the edge zone throughput lower bound. Without constraint of this factor, some edge zones may have very limited resources that negatively affects the whole network performance. The constraint made by this factor is given by:

$$
\sum_{i \in \mathcal{F}} A_{i}^{(u)} \geq B_{L}^{(u)}, \quad \forall u \in V .
$$

Edge spectrum partition constraint factor: $B_{U}^{(u)}$ is used to limit the maximum amount of subchannels allocated for edge zone. Different from $B_{L}^{(u)}$ that attempts to allocate more resources to edge zone when traffic load is light, $B_{U}^{(u)}$ provides more radio resource to center zone while traffic load is heavy. On the other hand, this factor is able to reserve resources for center zone to avoid negative effect on adjacent cell edge zones. As shown by edge zone allocation and transmit power constraints, a large usage of subchannels by a cell edge zone may result in scarcity of radio resources in adjacent cell edge zones. The constraint can be expressed by:

$$
\sum_{i \in \mathcal{F}} A_{i}^{(u)} \leq B_{U}^{(u)}, \quad \forall u \in V .
$$

The actual division of radio resources will be between the two bounds as Fig. 2 illustrates, where vertical axis $(P)$ represents power level and horizontal axis $(f)$ denotes frequency resource. The minimum amount of edge spectrum partition is denoted by the dark area on the left of the figure, and the minimum amount of center partition is denoted by the dark region on the right, which are limited by the two bounds. 


\section{D-FFR Algorithm}

There are three major steps in our scheme to form differentiated spectrum partitions in order to satisfy the constraints discussed in the previous section. We firstly construct a node weighted constraint graph, which reflects the constraint relationship among the cells in the OFDMA network. Then, we iteratively search the maximal independent node sets [9] and allocate subchannels to form spectrum partitions. The transmit power for different subchannels is sequentially determined according to usage situations.

Firstly, spectrum partitions for cell edge zones are considered in the network. In constructed graph $G(V, E)$, the nodes are assigned weights according to traffic loads, where edge zone traffic loads are of special interest. Given node $u \in V$, the node weight $w(u)$ is equivalent to the traffic load of edge zone. Independent node set, a subset of $V$ such that no two nodes in the subset are adjacent, can be found in graph $G(V, E)$. We iteratively search a maximal independent node set, a set which is not a subset of any other independent node set, to mitigate ICI and allocate subchannels for edge zones. The search of maximal independent node set is not only to find the maximal number of BSs reusing identical radio reuse but also to obtain a group of BSs desirable to schedule transmissions. To illustrate the proposed algorithm, we use the following definitions.

Definition 1: Given graph $G(V, E)$ and set $I$ formed by a subset of node set $V$, if a node $w$ is not in set $I$ but adjacent to a node $v$ in set $I$, we say $w$ is adjacent to set $I$.

Definition 2: Given graph $G(V, E)$ and set $I$ formed by a subset of node set $V$, we denote the set of all nodes in $V$ that are adjacent to set $I$, by $J(I)$, which is expressed by:

$$
J(I)=\{u \mid u v \in E, u \notin I, v \in I\} .
$$

From the definitions, it is obvious that $I \bigcap J(I)$ is empty. When we have a set $I$ and its adjacent node set $J(I)$, we can easily determine whether a node $u$ is independent of set $I$ or not. Given a node $u$ that is not in set $I \cup J(I)$ but adjacent to set $I \bigcup J(I)$, the node in $I \bigcup J(I)$ adjacent to node $u$ must be in set $J(I)$. This statement can be easily proved by contradiction. If $u$ is adjacent to a node in $I$, it will be in set $J(I)$, which is a contradiction to the assumption. We also can have such a node set $J(I \bigcup J(I))$ that any node in set $J(I \bigcup J(I))$ is adjacent to set $I \bigcup J(I)$.

Algorithm: At the beginning of the partition formation algorithm, independent node set $I$ is initiated as an empty set. It takes the node with the maximum weight as the first element and forms corresponding set $J(I)$. Set $J(I \cup J(I))$ is also sequentially formed. The algorithm then picks up node $u$ which has the maximum weight in $J(I \bigcup J(I))$, to add in set $I$. Thereafter, it updates sets $J(I)$ and $J(I \cup J(I))$ since a new node is added in $I$. Such procedures repeat until $J(I \bigcup J(I))=\varnothing$, which means that no more independent node exists in the graph excluding set $I$. The obtained feasible solution is denoted by $I^{*}$.

Our algorithm will examine the set weight, which is the accumulation on the weight of the elements in the set, to see whether $I^{*}$ is the heaviest one that we can find in a reasonable number of steps. The algorithm looks for a node $v, v \in V-I^{*}$ such that node $v$ has and only has an adjacent node $w, w \in I^{*}$. All such nodes that have not been selected in $I^{*}$ form a node set $L$. If such a node cannot be found, the independent node set $I$ is the maximal independent node set after the search process. Otherwise, the algorithm starts a process searching a heavier independent node set: It picks up the the maximum weight node from set $L$, for example, node $v$. Then, it adds $v$ in set $I^{*}$ and removes the adjacent node $w$ from $I^{*}$. After this, it searches the node that is not adjacent to any nodes in $I^{*}$. For a set of such nodes, it selects the node with the maximum weight and adds into set $I^{*}$. The search continues until no such a node can be found. The newly formed independent node set is used to compare with the previous one. This will be repeated, until all nodes in set $L$ has been visited. If a heavier set is found, it will be examined in the same way. Otherwise, it is terminated and outputs the maximal independent node set found so far.

The algorithm allocates available subchannels to the nodes in the maximal independent node set $I^{*}$. The node weighted constraint graph is then updated for next iteration. During the updating process, the weights of the nodes selected in the set are reduced since they constitute scheduled subchannel for edge spectrum partition. For the node in the set, if the weight is non-positive and the allocated resource is above the edge spectrum partition lower bound, the node and the corresponding edge(s) will be removed from the node weighted constraint graph. If allocated resource of node reaches the maximum allowed resource, i.e., edge spectrum partition constraint factor, the node and corresponding edge(s) are removed as well. The iteration terminates until the node weighted constraint graph is empty or all the resources have been used up. The center spectrum partition of each BS is determined by the complementary of edge spectrum partition.

Complexity: The computational complexity in determining all the nodes adjacent to set $I$ is no more than $\min \{6|I|,|V|-$ $|I|\}$ since the maximum degree of nodes is no more than six. Similarly, finding set $J(I \cup J(I))$ is no more than $\min \{6|J(I)|,|V|-|J(I)|\}$. To obtain the maximum weighted node in $J(I \cup J(I))$, the computational complexity is no more than $|J(I \cup J(I))|$. The cardinality of set $I$ increases from one but no more than $|V|$. The number of steps for all the operations is $O\left(n^{3}\right)$, where $n=|V|$. The procedures of searching whether there exists a higher weighted independent node set from set $J\left(I^{*}\right)$ and determining $J\left(I^{*}\right)$ from $G(V, E)$ are no more than $6\left|I^{*}\right|$ steps. To determine if the node in $J\left(I^{*}\right)$ has and only has one adjacent node in $I^{*}$, it takes no more than six steps. After finding such a node and exchange it with the corresponding node in $I^{*}$, the computational complexity in the rest procedures of finding a new independent set is $O\left(n^{3}\right)$. It performs at most $n$ times such exchange and search. Thus, the computational complexity is $O\left(n^{4}\right)$.

It should be noticed that the change of radio allocation is neither per-frame nor per-user based. It takes the long term radio usage conditions into account and the assignment will last relatively longer period. Therefore, the computation 


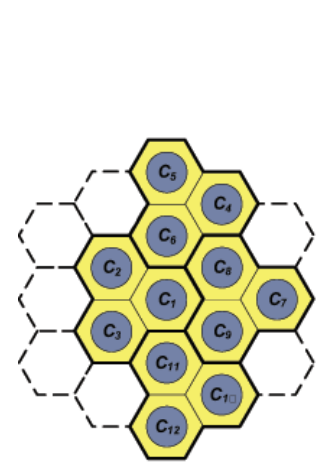

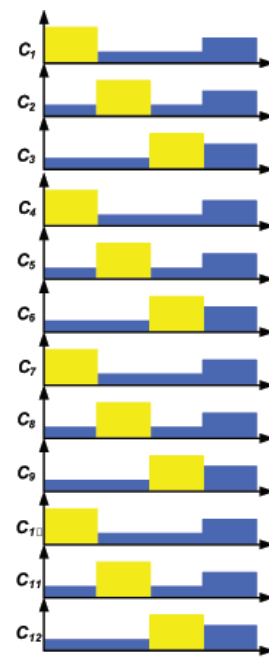

(b) Identical partition

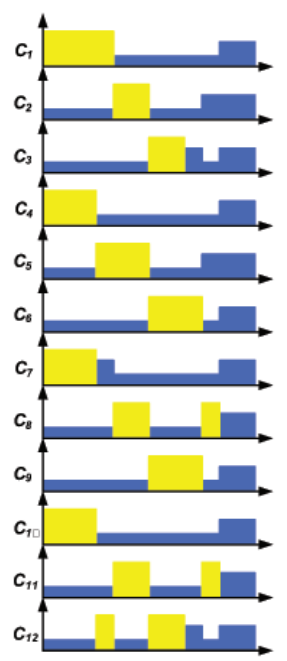

(c) Differentiable partition (a) Cells

Fig. 3: D-FFR example

complexity tolerance of our FFR scheme is much higher as compared to that of per-frame or per-user dynamic channel assignment.

Fig. 3 shows an example of D-FFR spectrum partitions and the compare with conventionally identical and continue partitions. In Figs. 3(b) and (c), horizontal axis denotes the transmit power and vertical axis denotes the radio spectrum, where the light (yellow) color denotes the edge spectrum partition and the dark (blue) color denotes the spectrum partition for center zone. Fig. 3(b) is the identical spectrum partition for the twelve cells shown in Fig. 3(a). We can see that no adjacent cell edge zone have overlapping radio resource, and each cell and zone has the same amount resource. Fig. 3(c) is the example of D-FFR having differentiated spectrum partitions, which has a larger edge spectrum partition for cell $C_{1}$ and larger center spectrum partition for cell $C_{3}$.

After spectrum partitions are determined, some residual spectrum capacity used by adjacent cells can be further utilized by the system. Assuming cell $u$ using a subchannel $i$ in its center zone, if $i$ is not used by adjacent cell edge zones, $u$ can use the subchannel with transmit power $P_{c 1}$ instead of $P_{c 2}\left(P_{c 1}>P_{c 2}\right)$. Every subchannel allocated to a cell's center zone needs to check the usage of the subchannel at the adjacent cell edge zones and then adjusts the transmit power, so as to maximize the enter zone throughput.

After these procedures, the spectrum partitions are generated and corresponding transmit powers are also determined for higher spectrum efficiency in dealing with various traffic loads.

\section{Performance Evaluation}

In this section, we investigate the D-FFR performance using extensive simulation. The configuration of simulation follows the suggestions in the IEEE $802.16 \mathrm{~m}$ evaluation methodology document [10], and is illustrated in Tab. I.

Fig. 4 compares the edge zone throughput with three different adjustment methods, where the arrangement of twelve

TABLE I: Simulation configuration

Cell Parameters

\begin{tabular}{ll}
\hline Number of Cells & 19 \\
Inter-site distance & $1500 \mathrm{~m}$ \\
\hline OFDMA parameters & \\
\hline Total Bandwidth & $30 \mathrm{MHz}$ \\
Carrier Frequency & $2.5 \mathrm{GHz}$ \\
Number of channels & 30 \\
Number of carriers per channel & 14 \\
\hline Channel Model & \\
\hline Path Loss $(\mathrm{dB})$ & $130.19+37.6 \log _{10}(d),(d$ in $k m)$ \\
\hline Power Control & \\
Cell-center Transmit Power 1 & $46 \mathrm{dBm}$ \\
Cell-center Transmit Power 2 & $40 \mathrm{dBm}$ \\
Cell-edge Transmit Power & $52 \mathrm{dBm}$ \\
Thermal Noise Density & $-174 \mathrm{dBm} / \mathrm{Hz}$ \\
\hline
\end{tabular}

cells is shown in Fig. 3(a). We increase the traffic load in the edge zone of cell $C_{1}$ (the center cell). The reason to adjust the center cell is to ignore the side effect. The edge zone of the center cell has the same ICI from all directions as long as MSs are uniformly distributed. To deal with the traffic demand increase demand at cell $C_{1}$ edge zone, "Soft FFR A AllCellAdj" has to adjust all cell edge spectrum partitions with the same configuration at the same reuse distance. "Soft FFR A -IdvCellAdj" only extends the edge spectrum partition of cell $C_{1}$. "Soft FFR A -IdvClstAdj" reduces the edge spectrum partition of cell $C_{2}$ to release radio resource for the edge spectrum partition of cell $C_{1}$. From Fig. 4, we see that the proposed scheme provides substantial improvement that increases the throughput with the traffic load. Other adjustment methods violate the constraints discussed previously. So, the edge zone throughput cannot be increased. It is even drastically decreased in "Soft FFR A -AllCellAdj". Our proposed D-FFR scheme considers the constraints and is able to achieve appropriate resource allocation with lower computational complexity.

Figs. 5 and 6 show the adjustment of radio division between the edge zone and the center zone in a single cell. We take the six cells around the center cell for observation, since they have similar ICI. We give an index number for each cell as $2,3,4,5,6$, and 7 (the center cell is numbered by 1). Then, we adjust one of the cells, i.e., cell 2. By decreasing the edge spectrum partition constraint factor, the edge zone throughput of cell 2 is decreased as expected. As shown in Fig. 6, the center zone throughput adaptively increases as well, because the scheme exploits the resource released by the edge zone. Due to the adaptivity in allocation, reduction in edge spectrum partition in one cell does not have much effect on other cell's throughput. The scheme algorithm adaptively adjusts the size and location of the partitions and avoids potential ICI caused by decreasing the edge spectrum partition at cell 2 . Thus, throughput of different cells and zones can be easily adjusted.

Figs. 7, 8, and 9 illustrate the network performance when the traffic load is not uniformly distributed in the cells, where $B_{U}(\cdot)$ in the figure denotes the setting of edge spectrum partition constraint factor. These figures illustrate the capabilities of our proposed scheme on radio resources not used by the edge zones of adjacent cells. Fig. 7 shows the overall throughput of the network when the traffic load at 


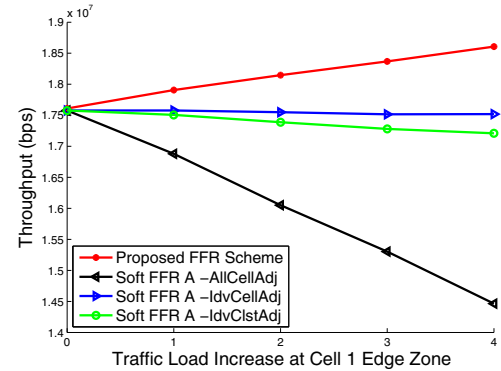

Fig. 4: Allocation adjustment

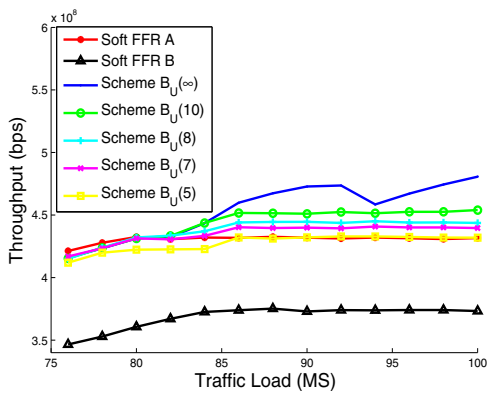

Fig. 7: Overall throughput

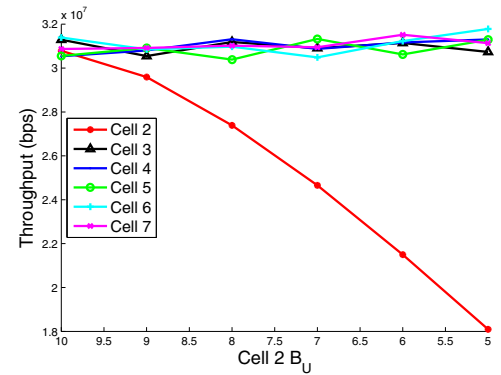

Fig. 5: Throughput in edge zone

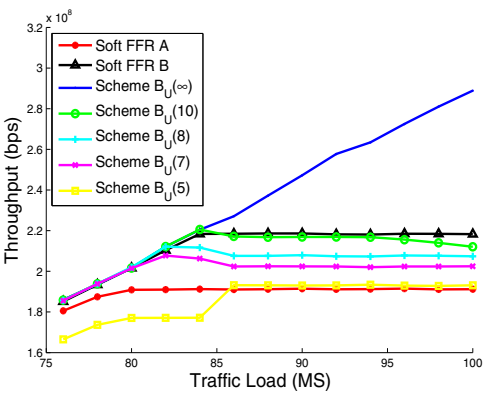

Fig. 8: Throughput in edge zones

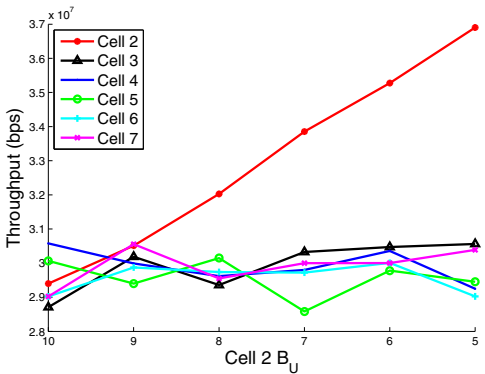

Fig. 6: Throughput in center zone

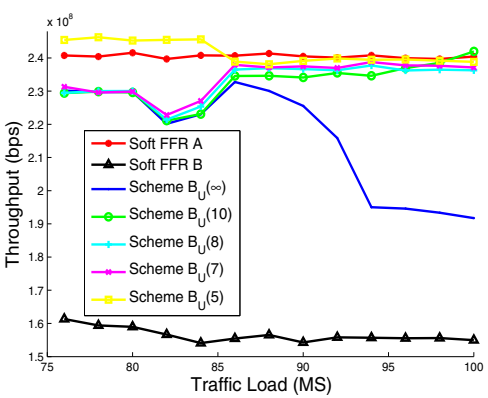

Fig. 9: Throughput in center zones the edge zone of the center cell is increased. While the traffic load increases, soft FFR A levels as it reaches its maximum resource allowed to the edge zone. Soft FFR B also reaches its maximum edge zone throughput at a later point. The proposed scheme can continuously increase the edge zone throughput due to its ability to increase the spectrum partition by using the subchannels not allocated by adjacent cells at the edge zones. Due to edge spectrum partition constraint factor, the throughput of D-FFR will level off at an upper bound as shown in Figs. 7 and 8. Given a higher edge spectrum partition factor, the edge zone throughput is also larger. The edge zone throughput curve without edge spectrum partition constraint can keep on increasing as shown in Fig. 8. The center zone throughput for the one without edge spectrum partition constraint decreases, as throughput increase of the edge zone starts hurting the throughput at the center zones of the cells. Therefore, appropriate setting of the factors enable differentiating spectrum partition to be within reasonable limits.

\section{CONCLUSION}

This paper studies the performance of FFR schemes, starting from the discussion on three major types of FFR schemes: hard FFR, soft FFR A, and soft FFR B. The analysis shows that non-uniform distributed ICI and traffic load require nonidentical frequency reuse. To support adaptive fractional radio reuse in a multi-cell OFDMA network, D-FFR scheme featured with differentiated and discrete partitions in a frequency band is proposed and analyzed in this paper. By considering ICI mitigation and radio resource allocation constraints, our proposed D-FFR scheme is able to optimally allocate radio resource in partitions and perform appropriate power control for uneven traffic loads to enhance the network performance. The simulation results show that our proposed D-FFR can adaptively perform differentiated spectrum partition for FFR while satisfying various constraints. Such mechanism can be utilized by current multi-cell OFDMA network, such as the IEEE 802.16 or the LTE, to improve spectrum efficiency and flexibility.

\section{REFERENCES}

[1] D. P. Agrawal and Q. Zeng, Introduction to wireless and mobile systems. Thomson Brooks/Cole Pacific Grove, CA, 2003.

[2] C. D. M. Cordeiro and D. P. Agrawal, Ad hoc \& sensor networks: theory and applications. World Scientific, 2006.

[3] J. G. Andrews, A. Ghosh, and R. Muhamed, Fundamentals of WiMAX: Understanding Broadband Wireless Networking. Prentice Hall PTR Upper Saddle River, NJ, USA, 2007.

[4] X. Wu, A. Das, J. Li, and R. Laroia, "Fractional power reuse in cellular networks," in Proceedings of the 44th Allerton Conference on Communication, Control, and Computing, 2006.

[5] "The draft IEEE 802.16m system description document," in IEEE 802.16 Broadband Wireless Access Working Group.

[6] E. Dahlman, H. Ekstrom, A. Furuskar, Y. Jading, J. Karlsson, M. Lundevall, and S. Parkvall, "The 3G long-term evolution-radio interface concepts and performance evaluation," in Vehicular Technology Conference, 2006.

[7] Q. Du and X. Zhang, "Resource allocation for downlink statistical multiuser QoS provisionings in cellular wireless networks," in Proceedings of IEEE INFOCOM, 2008.

[8] R. Rajkumar, C. Lee, J. Lehoczky, and D. Siewiorek, "Practical solutions for QoS-based resource allocation problems," in the 19th IEEE RealTime Systems Symposium, 1998.

[9] R. Diestel, Graph Theory. Springer, 2005.

[10] "Project $802.16 \mathrm{~m}$ evaluation methodology document (EMD)," in IEEE 802.16 Broadband Wireless Access Working Group. 